\title{
Some numerical methods for solving geodesic active contour model on image segmentation process
}

\author{
Maizatul Nadirah Mustaffa ${ }^{a}$, Norma Alias $^{\text {b, }}{ }^{,}$, Faridah Mustapha $^{a}$ \\ a Department of Mathematical Sciences, Faculty of Science Universiti Teknologi Malaysia, 81310 Johor Bahru, Malaysia \\ ${ }^{b}$ Centre for Sustainable Nanomaterials, Ibnu Sina Institute for Scientific and Industrial Research, Universiti Teknologi Malaysia, Skudai 81310, \\ Johor, Malaysia \\ * Corresponding author: norma@ibnusina.utm.my
}

\section{Article history}

Received 16 October 2017

Accepted 8 November 2017

Graphical abstract

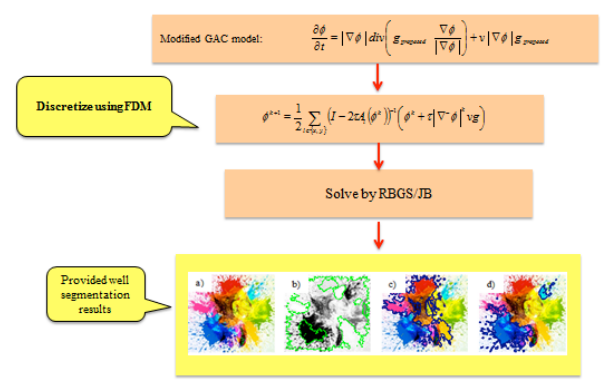

\begin{abstract}
In this paper, we present an edge-based image segmentation technique using modified geodesic active contour model to detect the desired objects from an image. The stopping function of the proposed model has been modified from the usual geodesic active contour model. The modified geodesic active contour model is discretized using finite difference method based on the central difference formula. Then, some numerical methods such as RBGS and Jacobi methods are used for solving the linear system of equation. The accuracy and effectiveness of the proposed algorithm have been illustrated by applied to different images and some numerical methods.
\end{abstract}

Keywords: Image segmentation, color, geodesic active contour, numerical methods

(C) 2017 Penerbit UTM Press. All rights reserved

\section{INTRODUCTION}

Recently, geodesic active contour has been widely used as a tool for image segmentation process and until now, has been growing significantly [1]. Segmentation can be classified as follows: region based, edge based, threshold, feature based clustering and model based. The basic idea of geodesic active contour is to evolve color detection as a stopping operator to get desired objects. Geodesic active contour is based on gradient to detect boundary [1]. The geodesic active contour can segment all objects in the image. However it cannot detect desired objects of color. To overcome this problem, we proposed a new stopping function in which this stopping criterion is based on gradient, edge and color information of the image.

A geodesic active contour is part of the active contour that uses the gradient to find the edge of a feature and detect the contours. Many improvements of geodesic active contour have been made by researchers for solving the weakness of the model discretization. The main weakness of this model is not linear in terms of equations and some dependent parameters [2]. This may cause inefficient implementation on convergence and accuracy of the algorithm.

To overcome the non-linearity of geodesic active contour, [2] adapted a numerical technique based on additive operator splitting scheme. Goldenberg et al. [2] has proved the improvement model obtained the numerical consistency and computational efficiency of the geodesic active contour. Then an additive operator splitting scheme is employed to further improve the efficiency of the flow-field smoothing. Additive operator splitting scheme can be implemented by recursive filtering. The reparability nodes allow a straightforward implementation on any dimension region. Weickert et al. [6] proved that the additive operator splitting scheme was ten times more efficient than the explicit scheme under typical accuracy. The advantage of this image analysis can identify the unexplored region while providing imaging, resolving unanswered by the integrated modeling, transmission images better than reflection images.

This paper is organized as follows. In section 2 we describe our modified geodesic active contour based on gradient, edge and color information of the color images. Section 3 discusses the numerical implementation. Our experimental results and discussion are given in Section 4. Section 5 concludes the paper.

\section{MODIFIED GEODESIC ACTIVE CONTOUR}

Geodesic active contour were introduced by Casellas et al [3] as a segmentation framework, derived from energy-based snake active contours and performing contour extraction via the computation of geodesic. We use the GAC model to detect the edges of desired object. The classical geodesic active contour model in partial differential equation (PDE) formulation is

$$
\begin{gathered}
\frac{\partial \phi}{\partial t}=|\nabla \phi| \operatorname{div}\left(g \frac{\nabla \phi}{|\nabla \phi|}\right)+v|\nabla \phi| g \\
\text { on } \Omega \times(0, \infty) \\
\phi(x, 0)=\phi_{0}(x) \text { on } \Omega
\end{gathered}
$$


where $\phi$ denotes the level value of set, $v$ is parametric active contour of snake, $\nabla$ is the gradient operator, $g$ as a stopping function, $\Omega$ is the image domain and $|\nabla \phi|$ is to controls the interior and exterior of contour. There few steps involve in geodesic active contour process. At the first step, any curve that closed to the targeting object will be initialized. The second step is iterative process. During the iterative process, the active contour deforming and moving towards the desired object boundary by various forces that control the shape and location of the contour within the image. In the end, it completely shrink-wraps around the object. The stopping function approaches 0 whenever a contour line closes to the edges with high gradient magnitude. With low gradient magnitude, it reaches 1 in flat image areas [7]. In order to raise geodesic active contour model performance on multiple objects and complex background, we proposed new stopping function of geodesic active contour. The idea of modifying the stopping function was inspired by Ling. P [1] and Goldenberg [2].

$$
g_{\text {Ling.P }}=\frac{1}{1+\Lambda^{2}\left(G_{\sigma} * \phi\right)}
$$

and

$$
g_{\text {Goldenberg }}=\frac{1}{1+K \Lambda^{2}}
$$

By integrating both stopping function defined in eq (3) and (4), we get new stopping function formulation.

$$
g_{\text {proposed }}=\frac{1}{1+K \Lambda^{2}\left(G_{\sigma} * \phi\right)}
$$

where $K>0$ is the contrast factor, $\Lambda$ is the largest eigenvalue of the metric tensor in the $\{x, y, R, G, B\}$ space [1], $\left(G_{\sigma} * \phi\right)$ is the convolution of the image $\phi$ with the Gaussian $G_{\sigma}=\sigma^{1 / 2} \exp \left(-\left|x^{2}+y^{2}\right| / 4 \sigma\right) \quad[1]$ and $\sigma_{\text {is a parameter. }}$

Then, the formulation becomes

$$
\frac{\partial \phi}{\partial t}=|\nabla \phi| \operatorname{div}\left(g_{\text {proposed }} \frac{\nabla \phi}{|\nabla \phi|}\right)+v|\nabla \phi| g_{\text {proposed }} \text { (6) }
$$

Our proposed stopping function improves the classical stopping function by adding the color information of an image. Thus, the curve will stop near to desired color object(s). We used finite difference scheme to discretized eq (6) and linearized using additive operator splitting scheme $[4,5]$. To derive a numerical algorithm of this model, we consider the discretization of space and time. For time discretization, $\tau$ is denoted as time step size and $t_{k}=k \tau$, where $k \in N_{0}$. Then, the evolution in $x$ and $y$ direction is represent respectively in eq (7) and eq (8)

$$
\begin{aligned}
& \left(\begin{array}{c}
1+2 \tau \frac{2|\nabla \phi|_{i, j}^{k}}{\left(\frac{|\nabla \phi|}{g_{\text {proposed }}}\right)_{i, j-1}^{k}+\left(\frac{|\nabla \phi|}{g_{\text {proposed }}}\right)_{i, j}^{k}} \\
+2 \tau \frac{2|\nabla \phi|_{i, j}^{k}}{\left(\frac{|\nabla \phi|}{g_{\text {proposed }}}\right)_{i, j+1}^{k}+\left(\frac{|\nabla \phi|}{g_{\text {proposed }}}\right)_{i, j}^{k}}
\end{array}\right)^{k+1, j} \\
& -2 \tau \frac{2|\nabla \phi|_{i, j}^{k}}{\left(\frac{|\nabla \phi|}{g_{\text {proposed }}}\right)_{i, j-1}^{k}+\left(\frac{|\nabla \phi|}{g_{\text {proposed }}}\right)_{i, j}^{k}} x_{i, j-1}^{k+1} \\
& -2 \tau \frac{2|\nabla \phi|_{i, j}^{k}}{\left(\frac{|\nabla \phi|}{g_{\text {proposed }}}\right)_{i, j+1}^{k}+\left(\frac{|\nabla \phi|}{g_{\text {proposed }}}\right)_{i, j}^{k}} x_{i, j+1}^{k+1}
\end{aligned}
$$

$=\phi_{i, j}^{k}+\tau|\nabla \phi|_{i, j}^{k} v g_{\text {proposed }}$

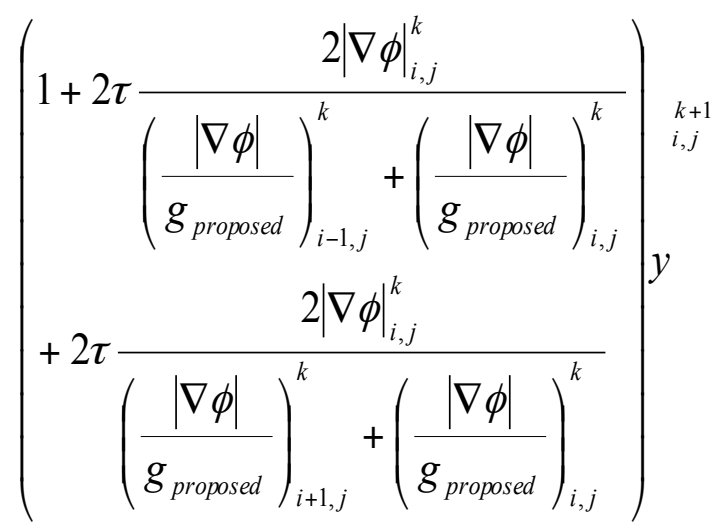

$-2 \tau \frac{2|\nabla \phi|_{i, j}^{k}}{\left(\frac{|\nabla \phi|}{g_{\text {proposed }}}\right)_{i-1, j}^{k}+\left(\frac{|\nabla \phi|}{g_{\text {proposed }}}\right)_{i, j}^{k}} y_{i-1, j}^{k+1}$

$-2 \tau \frac{2|\nabla \phi|_{i, j}^{k}}{\left(\frac{|\nabla \phi|}{g_{\text {proposed }}}\right)_{i+1, j}^{k}+\left(\frac{|\nabla \phi|}{g_{\text {proposed }}}\right)_{i, j}^{k}} y_{i+1, j}^{k+1}$

$=\phi_{i, j}^{k}+\tau|\nabla \phi|_{i, j}^{k} v g_{\text {proposed }}$ 
The solution of the linear system of equation above can be done using some numerical approach that has been described in the next section. The computational platform is based on Intel ${ }^{\circledR}$ CoreTM Duo Processor Architecture with MATLAB version R2011a and will be used to select and process the image. Then from the information of the image, the pixels of image will be decomposed by additive operator splitting in horizontal and vertical directions.

\section{NUMERICAL IMPLEMENTATION}

A linear system of equations is a collection of one or more linear equations involving some variables [8]. It can be expressed in matrix form as,

$$
A \phi=f
$$

where

$$
A=\left[\begin{array}{cccc}
a_{11} & a_{12} & \ldots & a_{1 n} \\
a_{21} & a_{22} & \ldots & a_{2 n} \\
\vdots & \vdots & \ddots & \vdots \\
a_{n 1} & a_{n 2} & \ldots & a_{n n}
\end{array}\right] \phi=\left[\begin{array}{c}
\phi_{1} \\
\phi_{2} \\
\vdots \\
\phi_{n}
\end{array}\right] f=\left[\begin{array}{c}
f_{1} \\
f_{2} \\
\vdots \\
f_{n}
\end{array}\right]
$$

where $A$ is the coefficient matrix, $\phi$ corresponds to vector of unknowns and $f$ is the right hand side vector. Red Black Gauss Seidel (RBGS) and Jacobi (JB) method start the iteration to solve the linear system for both directions until the convergent criterion is satisfied. The numerical result then will be analysed in term of time execution, number of iterations, maximum error, and root mean square error (RMSE).

\section{Red Black Gauss-Siedel (RBGS) Method}

Red-Black Gauss-Seidel (RBGS) method is an iterative method that contain two-subdomain which are $\Omega^{R}$ and $\Omega^{B}$. Thus, there is communication between these two-subdomains. Red point depends on the black point, and vice-versa. The loop starts by computing the odd points, from the bottom left and then going up to the next row and so on. When the all odd points are finished, do the black ones. The red black grid is illustrated in Fig.1.

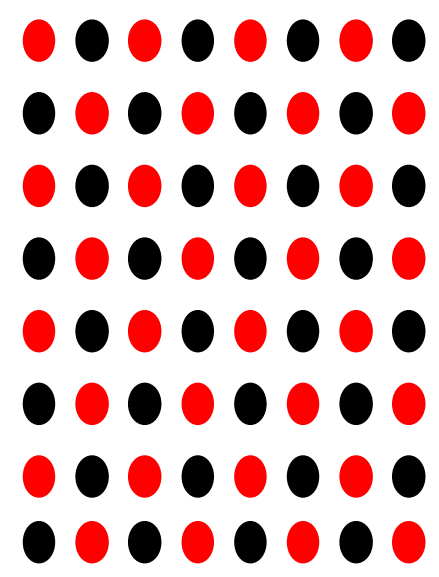

Fig. 1 The grid for red and black points.
The algorithm of this method is shown as follows:

\section{Algorithm 1: RBGS method}

for $t_{i}=1$ : TIMESTEP

for $i=1: n$ (Grid Calculation $\Omega^{R}$ )

$\phi_{i}^{(k+1)}=\frac{1}{a_{i i}}\left(f_{i}-\sum_{j=1}^{i-1} a_{i j} \phi_{j}^{(k+1)}-\sum_{j=i+1}^{n} a_{i j} \phi_{j}^{(k)}\right)$

$i=1,3,5, \ldots, n$

end

for $i=2: n$ (Grid Calculation $\Omega^{B}$ )

$\phi_{i}^{(k+1)}=\frac{1}{a_{i i}}\left(f_{i}-\sum_{j=1}^{i-1} a_{i j} \phi_{j}^{(k+1)}-\sum_{j=i+1}^{n} a_{i j} \phi_{j}^{(k+1)}\right)$

$i=2,4,6, \ldots, n$

end

err $=\left|\phi^{k+1}-\phi^{k}\right|$

if err $<$ tol

break

end

end

\section{Jacobi Method}

Jacobi method is a classical iterative method and was attributed to Carl Jacobi. It computes the value of $\phi$ for each component with respect to $x \quad$ and $y$. Matrix $A$ decomposes into $A=D-L-U$ which lead to the vector form of Jacobi iteration.

$$
\phi^{k+1}=D^{-1}(L+U) \phi^{k}+D^{-1} f, k=0,1, \ldots
$$

Equation (8) can be shown in discrete equation as

$$
\phi_{i}^{(k+1)}=\frac{1}{a_{i i}}\left(f_{i}-\sum_{j=1}^{n} a_{i j} \phi_{j}^{k}\right) \quad i=1,2, \ldots, n
$$

The calculation of this method is shown by the algorithm below:

\section{Algorithm 2: Jacobi method}

for $t_{i}=1$ : TIMESTEP

$$
\begin{aligned}
& \text { for } i=1: n \\
& \phi_{i}^{(k+1)}=\frac{1}{a_{i i}}\left(f_{i}-\sum_{j=1}^{n} a_{i j} \phi_{j}{ }^{k}\right) \quad i=1,2, \ldots, n \\
& \text { end } \\
& \text { err }=\left|\phi^{k+1}-\phi^{k}\right| \\
& \text { if err < tol } \\
& \text { break } \\
& \text { end } \\
& \text { end }
\end{aligned}
$$

\section{EXPERIMENTAL RESULT AND DISCUSSION}

Modified geodesic active contour has been tested in two difference color images to show the improvements over the classical geodesic active contour model [4,5]. The result will be presented based on visualization and numerical analysis. 

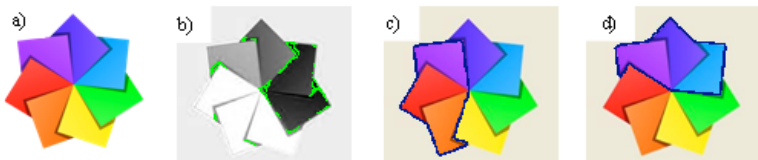

Fig. 2 Result for detection of the classical GAC (b), modified GAC (c) and (d) for $K=0.35$ on image 1 .
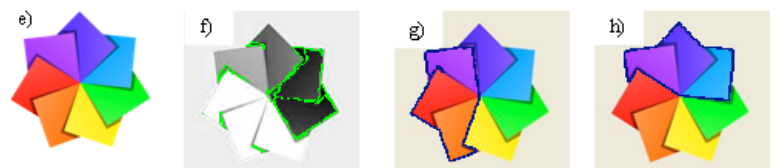

Fig. 3 Result for detection of the classical GAC ( $f$ ), modified GAC (g) and $(\mathrm{h})$ for $K=0.5$ on image 1 .
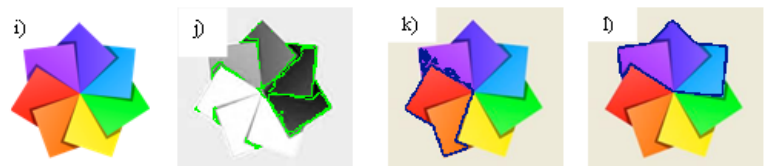

Fig. 4 Result for detection of the classical GAC (j), modified GAC (k) and $(\mathrm{I})$ for $K=0.65$ on image 1 .
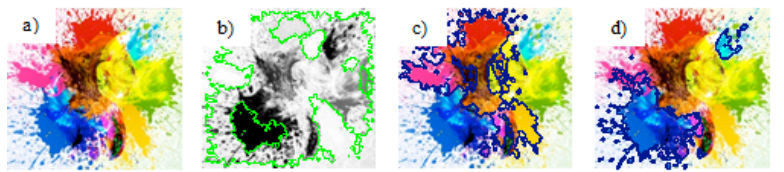

Fig. 5 Result for detection of the classical GAC (b), modified GAC (c) and (d) for $K=0.35$ on image 2 .
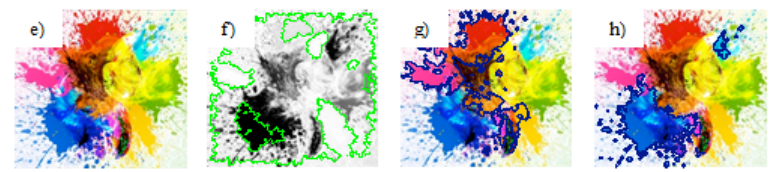

Fig. 6 Result for detection of the classical GAC (f), modified GAC (g) and $(h)$ for $K=0.5$ on image 2 .
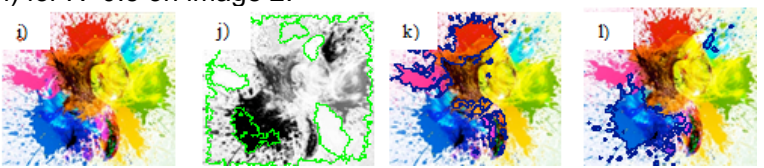

Fig. 7 Result for detection of the classical GAC (j), modified GAC (k) and $(I)$ for $K=0.65$ on image 2 .

The proposed modified geodesic active contour model has been applied to real color images. In Fig. 2 until Figure 4, we demonstrate the edge detection on simple image and Fig. 5 until Fig. 7, we use an image with complex background. The algorithm is implemented by MATLAB version R2011a. In each experiment, the modified geodesic active contour model is initialized by the function $\phi_{0}(x)$ defined in eq (2) with $K=0.35,0.5$ and 0.65 . We can find in Fig. 2 until Fig. 7 with $200 \times 200$ pixel, classical geodesic active contour only can detect based on the gradient of images and cannot detect the desired color of image. However, our modified model able to detect desired color for example in Fig. 5c, we detect red color paper and Fig.5d detect the blue color paper. This shows that our proposed model is more robust when the object boundaries are complex. From Table 1, our proposed model shows the best performance which is more accurate and performs faster than classical GAC model. The proposed model provides the less number of iteration and time of execution to converge. The accuracy method is based on the RMSE. If the RMSE is lowesst, then it will represents the most accurate method. Absolute error also can describe the accuracy of each method. From Table 1, it is shown that the proposed model is the most accurate detection method due to the smallest value of RMSE. In addition, from the obtained results, RBGS method is the alternative method with the lowest time of execution, number of iteration and RMSE.

\section{CONCLUSION}

In this paper, a modified geodesic active contour model is proposed in order to detect and segment the desired color of an image. The proposed model has been modified on its stopping function to the final PDE formulation. The new stopping function, help to obtain and detect the desired color object and raise the performance on images with complex background. Thus, the new modified geodesic active contour model has been discretized using finite difference method. RBGS method and Jacobi method is used to solve the linear system. Numerical results have shown that RBGS method as superior method than JB method. Finally, based on the RMSE, our modified geodesic active contour model is more robust to detect color object with respect to weak edges and complex background.

Table 1 Numerical analysis for different methods.

\begin{tabular}{|c|c|c|c|}
\hline Model & & $\begin{array}{c}\text { Proposed } \\
\text { Model }\end{array}$ & $\begin{array}{l}\text { Classical } \\
\text { GAC Model }\end{array}$ \\
\hline \multirow{3}{*}{$\begin{array}{l}\text { No. of } \\
\text { iterations }\end{array}$} & RBGS & 59 & 126 \\
\hline & JACOBI & 114 & 245 \\
\hline & $\%$ & 48.25 & 48.57 \\
\hline \multirow{3}{*}{$\begin{array}{c}\text { Time } \\
\text { Execution } \\
\text { (s) }\end{array}$} & RBGS & 0.7023 & 1.5275 \\
\hline & JACOBI & 1.0056 & 2.2558 \\
\hline & $\%$ & 30.16 & 32.29 \\
\hline \multirow{3}{*}{$\underset{\text { Error }}{\text { Maximum }}$} & RBGS & $8.4984 \mathrm{e}-011$ & $9.8287 e-11$ \\
\hline & JACOBI & $9.9856 \mathrm{e}-11$ & $9.8448 \mathrm{e}-11$ \\
\hline & $\%$ & 14.89 & 0.16 \\
\hline \multirow{3}{*}{ RMSE } & RBGS & $1.7503 e-012$ & $2.7105 e-11$ \\
\hline & JACOBI & $1.7809 \mathrm{e}-12$ & $3.0225 e-11$ \\
\hline & $\%$ & 1.72 & 10.39 \\
\hline
\end{tabular}

\section{ACKNOWLEDGEMENT}

This work was financially supported by the Universiti Teknologi Malaysia, Ministry of High Education (MOHE), STEM Grant with vote no. A. J091002.5600.07397.

\section{REFERENCES}

[1] Ling, P., Chaomin, S., Fang, L., Jinsong, Fan. 2007. A variational formulation for segmenting desired objects in color images. Image and Vision Computing. 25(9), Issue 9, 1414-1421.

[2] Goldenberg, R., Kimmel, R., Rivlin, E., Rudzsky, M. 2001. Fast geodesic active contours. IEEE Transactions Image Processing. 10, 1467-1475.

[3] Caselles, V., Kimmel, R., and Sapiro, G. 1997. Geodesic active contour. International Journal of Computer Vision. 22(1), 61-79.

[4] Alias, N., Mustaffa, M. N., Md. Zubaidin, Z., Saipol, H. F., Che Abd. Ghani, A. 2014. The chronology of geographic information system image processing: A case study for land changes at Nusajaya, Iskandar Malaysia. Advanced Science Letters. 20(2), 435-438(4).

[5] Md. Zubaidin, Z. 2013. Alternating group explicit method for edge detection on brain and breast tumuor images. Thesis Master of Science. Universiti Teknologi Malaysia, Skudai.

[6] Weickert, J., ter Haar Romeny, B. M., and Viergever, M. A. 1998. Efficient and reliable schemes for nonlinear diffusion filtering. IEEE Transactions on Image Processing. 7(3), 398-410.

[7] Kuhne, G., Weickert, J., Beir, M., and Effelsberg, W. 2002. Fast implicit active contour models. Proceedings of the $24^{\text {th }}$ DAGM Symposium on Pattern Recognition. 16-18 September 2002. Zurich, Switzerland: Springer-Verlag, 133-140.

[8] Che Rahim, C. T. 2009. Numerical Method: Algorithm and Matlab Programming. Third Edition. Universiti Teknologi Malaysia, Johor. 